\title{
Evaluation of different normalisation procedures for calculation of the standardised uptake value in therapy response monitoring studies
}

Running head: Evaluation of different normalisation procedures

Dennis Vriens, MD; Lioe-Fee de Geus-Oei, MD, PhD*; Hanneke W. van Laarhoven, MD, $\mathrm{PhD}^{\dagger}$; Johanna N.H. Timmer-Bonte, MD, PhD ${ }^{\dagger}$; Paul F.M. Krabbe, PhD ${ }^{\S}$; Eric P. Visser, PhD*; Wim J.G. Oyen, MD, PhD*

*Dept. of Nuclear Medicine, ${ }^{\dagger}$ Dept. of Medical Oncology, ${ }^{\dagger}$ Dept. of Pulmonary Diseases; ${ }^{\S}$ Dept. of Epidemiology, Biostatistics \& Health Technology Assessment.

Radboud University Nijmegen Medical Centre, Nijmegen, the Netherlands

Correspondence:

D. Vriens, MD

Department of Nuclear Medicine (internal postal code 444)

Radboud University Nijmegen Medical Centre

P.O. Box 9101, 6500 HB Nijmegen, the Netherlands

Phone: +31-24-3614048, Fax: +31-24-3618942

E-mail: D.Vriens@nucmed.umcn.nl

Word count text body: 3,299 


\section{Abstract}

Objectives: The aim of this prospective study was to assess the influence of different normalisation procedures on relative changes in standardised uptake values (SUV) of FDGPET for the assessment of chemotherapy response in patients with colorectal carcinoma 5 (CRC) and non-small cell lung carcinoma (NSCLC). Methods: In 97 patients with CRC $(n=48)$ and NSCLC $(n=49)$ FDG-PET was performed before and during the course of chemotherapy. Relative changes in SUV $(\Delta \mathrm{SUV})$ were determined after correction for injected dose and bodyweight, lean body mass, body surface area or a combination of bodyweight and plasma glucose. The predictive value for overall (OS) and progression free survival (PFS) with respect to the different normalised $\Delta$ SUVs was assessed. Results: In both CRC and NSCLC no differences were seen in the degree of change between the four SUV-normalisations during chemotherapy. Cox regression analysis for OS showed significant hazard ratios of 1.14-1.16 per ten percent SUV-change in CRC and 1.10-1.13 in NSCLC and for PFS hazard ratios of 1.15 per ten percent $\triangle$ SUV-change in CRC and 1.101.12 in NSCLC. Conclusions: Relative change of SUV is a strong predictor for survival in both CRC and NSCLC. None of the four normalisation methods showed statistical advantage over the other. Therefore, simplifying the methods for analysis of FDG-PET data can improve the incorporation of FDG-PET in clinical treatment-response monitoring and may facilitate application in multicentre trials.

Keywords: Colorectal carcinoma - Non-small cell lung carcinoma - $\left[{ }^{18} \mathrm{~F}\right] \mathrm{FDG}$ - Positron emission tomography - chemotherapy - Standardised uptake value - SUV - therapy monitoring - survival 
Functional imaging by ${ }^{18} \mathrm{~F}$-2-fluoro-2-deoxy-D-glucose positron emission tomography (FDG-PET) has an established role in the standard care of patients with both colorectal carcinoma (CRC) and non-small cell lung carcinoma (NSCLC). Interest in the application of FDG-PET for prediction and evaluation of tumour response to therapy is growing. When using morphological imaging techniques such as computed tomography $(\mathrm{CT})$ or magnetic resonance imaging (MRI) it may be difficult to reliably distinguish between necrosis, scar tissue and recurrent or residual tumour in CRC [1-3] and NSCLC [4]. Furthermore, metabolic alterations in tumour cells, indicative of tumour response to therapy, may arise earlier than changes in size [5]. Moreover, some new antitumour therapies are cytostatic rather than cytoreductive. Therefore success of treatment cannot reliably be measured on morphologic imaging modalities alone. Early detection of tumour progression during chemotherapy can prevent unbeneficial and potentially harmful treatment and provides the opportunity to modify treatment at an early treatment stage.

In CRC [6] as well as NSCLC [7-9] a significant prognostic value for overall 40 survival (OS) and progression-free survival (PFS) by (semi)quantitative measures of FDGPET data analysis has been shown. Also, the response to chemotherapy, evaluated as relative change in FDG-PET-assessed tumour metabolism in CRC [10,11] and NSCLC $[9,12]$, proved to be significantly associated with OS and PFS. In these studies it was suggested that less demanding semi-quantitative parameters such as the standardised uptake value (SUV) might perform as well as complex dynamic imaging protocols necessary for Patlak analysis $[11,13]$ which would facilitate broad introduction in clinical practice by improving patient compliance. Another advantage of the SUV is that it can be calculated from static, whole-body FDG-PET studies, which depict all metastases. In quantitative 
dynamic scans only one axial field of view $(15-20 \mathrm{~cm})$ can be studied which could exclude metastases which respond differently to therapy [11].

The present study aims at further standardisation, validation and simplification of the methods necessary for metabolic response assessment. For this purpose four distinct normalisation methods for relative changes in SUV ( $\triangle \mathrm{SUV})$ were evaluated in two patient populations (CRC and NSCLC). SUVs were determined from scans performed on one bed position only, since this study was part of a larger project, which included dynamic acquisition. This single axial field of view was chosen to include as many lesions as possible, based on the baseline whole-body staging FDG-PET, acquired at an earlier time point.

. Overall and progression free survival were used as outcome measures. For 60 absolute SUV measurement the different normalisation procedures are already extensively compared in former studies [14], of which some seem superior to others $[13,15]$ when compared to full parameter pharmacokinetics by nonlinear regression. These studies, however, address the prognostic value of SUV by its absolute value. In contrast, in the present study, the relative value will be examined, which is of importance in therapy 


\section{Patients and methods}

\section{Patient eligibility criteria}

Patients eligible for the FDG-PET chemotherapy response monitoring studies on CRC [11] and NSCLC [12] were included in the present study. In all patients, treatment decisions were based on current guidelines and made by a multidisciplinary team including medical oncologists, oncological and cardio-thoracic surgeons, pulmonologists, radiation oncologists, pathologists, radiologists and nuclear medicine physicians. All clinicians were blinded to the results of the FDG-PET scans. The study was approved by the Institutional

75 Review Board of the Radboud University Nijmegen Medical Centre and written informed consent was obtained from each patient.

\section{FDG-PET}

FDG-PET was performed at baseline and after two months of treatment (CRC) or after the second or third cycle of chemotherapy (NSCLC), depending on the chemotherapy regimen. Patients were fasted for at least $6 \mathrm{~h}$ before imaging. Intake of sugar-free liquids was permitted. A dose of $\sim 200 \mathrm{MBq}$ FDG (Covidien, Petten, the Netherlands) was injected intravenously. All scans were acquired between 40 and 50 minutes post injection on an ECAT-EXACT47 FDG-PET scanner (Siemens/CTI, TN, USA) in septa-extended (2dimensional) mode. The position of the patient in the scanner's field of view (162 mm in 47 planes) was based on whole-body FDG-PET and CT scans performed for routine clinical work-up, including as many measurable tumour lesions as possible. Only one field of view was scanned, since this study was part of a larger project, which included dynamic scanning $[11,12]$. A 20 -min transmission scan was performed, using the internal ${ }^{68} \mathrm{Ge} /{ }^{68} \mathrm{Ga}$ sources,

90 to correct for photon attenuation, the duration of which was chosen to provide a higher signal-to-noise ratio. The emission and transmission sinograms were corrected for randoms 
and decay. Scatter correction based on measured scatter fractions as implemented in the ECAT 7.2.1. software for 2D reconstructions was used. Attenuation-corrected images were reconstructed in $128 \times 128 \times 47$ matrices using filtered backprojection with a Gaussian filter of $4 \mathrm{~mm}$ full width at half maximum (FWHM). This resulted in voxels of $3.432 \mathrm{~mm} \mathrm{x}$ $3.432 \mathrm{~mm} \times 3.375 \mathrm{~mm}$ and a spatial resolution of $6 \mathrm{~mm}$ FWHM in the reconstructed images.

FDG-PET scans were evaluated semi-quantitatively by standardised uptake value (SUV) analysis. Tumour regions of interest (ROI) were obtained semi-automatically using a threshold of $50 \%$ of the maximum pixel value within the lesion. Four different SUVs, based on injected dose and bodyweight $\left(\mathrm{SUV}_{\mathrm{BW}}\right)$, lean body mass $\left(\mathrm{SUV}_{\mathrm{LBM}}\right)$, body surface area $\left(\mathrm{SUV}_{\mathrm{BSA}}\right)$ and a combination of bodyweight and plasma glucose $\left(\mathrm{SUV}_{\mathrm{BW}+\mathrm{G}}\right)$ were calculated (table 1) [14]. The injected dose was calculated by subtraction of the residual ${ }^{18} \mathrm{~F}$-activity of the infusion system from the ${ }^{18} \mathrm{~F}$-activity delivered by the laboratory, corrected for decay to time of injection: $I D=\left(D D \cdot e^{-\lambda \cdot(t-\tau)}\right)-\left(R D \cdot e^{-\lambda \cdot(t-\theta)}\right)$ where DD is 105 the delivered dose, $\mathrm{RD}$ is the residual dose, $\lambda$ is the decay constant of ${ }^{18} \mathrm{~F}\left(=0.006314 \mathrm{~min}^{-}\right.$ $\left.{ }^{1}\right),(t-\tau)$ is the time interval between delivery and injection and $(\mathrm{t}-\theta)$ is the (negative) time interval between activity measurement of the infusion system and injection.

When multiple lesions were quantified in one patient, a patient's mean SUV was calculated weighting every lesion by its volume by the formula:

110

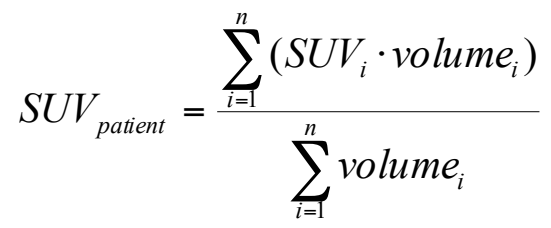

The relative change in SUV ( $\triangle \mathrm{SUV}$ ) between the baseline and second FDG-PET was calculated $\left(\triangle \mathrm{SUV}=\left[\mathrm{SUV}_{\text {follow-up }}-\mathrm{SUV}_{\text {baseline }}\right] \cdot \mathrm{SUV}_{\text {baseline }}{ }^{-1} \cdot 100 \%\right)$. All lesions which were completely visible in the FoV were included. There was no maximum to the number of lesions per patient. 


\section{Clinical Follow-Up}

During and after treatment, patients were followed-up with clinical- and radiological examination and laboratory tests at regular intervals. Morphologic tumour response was routinely evaluated according to Response Evaluation Criteria in Solid Tumours (RECIST) [16] without knowledge of the results of the FDG-PET studies. These criteria define progression as a $20 \%$ increase in the sum of longest diameters of target lesions or the appearance of new lesion [16]. When disease progression was suspected or proven, patients were always restaged by the previously mentioned multidisciplinary team. The date of local or distant progression was defined as the earliest date at which disease progression was confirmed, either clinically or by imaging or biopsy.

In patients who were progression free at the closeout date (April 2008) or who had died from any non-tumour related cause, the time to progression was censored at that date. Overall survival was measured from the date of the baseline FDG-PET scan to the date of death. In patients who were alive at the closeout date, survival was censored at that date.

\section{Statistical Analysis}

Normality of the data was assessed by the Shapiro-Wilk statistic. For normal distributions mean ( \pm standard deviation) are presented and the paired t-test was used for comparison. For

135 non-normal distributions, median (interquartile range, IQR) are presented as measures for central tendency and dispersion and the Wilcoxon signed ranks test was used for comparison. Comparing medians between dependent groups, Friedman two-way analysis of variance (ANOVA) by ranks was performed. The different normalised $\triangle$ SUVs were compared using a threshold for significant difference of $20 \%$. In studies comparing the testretest characteristics of FDG-uptake a test-to-test variability of $15-20 \%$ is found, meaning that a change in SUV between $\sim-20 \%$ to $\sim+20 \%$ is within the reproducibility limits of the test $[17,18]$. 
Overall survival (OS) and progression free survival (PFS) served as the standard of reference. Death of any cause was defined as an event in OS analysis. Survival time was defined as the time between first FDG-PET and event (death or progression). Kaplan-Meier analysis was performed to determine median survival. Dichotomisation was performed at the median metabolic response and strata were compared by the log rank test. Cox's proportional hazards model was used to assess the predictive value of response evaluation with FDG-PET, as expressed in the $\triangle$ SUVs between the FDG-PET at baseline and followup. Hazard ratios (HR) are presented with their 95\%-confidence interval (CI, Wald's $\chi^{2}$ test) together with the ratio in mean survival (median ratio). Finally, to investigate how a prognosis-driven threshold (as contrast to metabolic response-driven threshold) might prove of additional value, a cut-off for $\Delta \mathrm{SUV}$ was determined for which $>90 \%$ of the patients show 1-year OS.

155 Analysis was performed with the Statistical Package for Social Sciences (SPSS ${ }^{\text {) }}$ ) version 16.0.2 for Mac (SPSS Inc, Chicago, Illinois, USA) and GraphPad Prism 5.0a for Mac (GraphPad Software Inc, La Jolla, California, USA). Statistical tests were based on a two-sided significance level and the level of significance was set at $\mathrm{p}=0.05$ for all tests.

\section{Results}

\section{Patient characteristics}

One hundred and twenty consecutive eligible patients were included in this prospective study (61 advanced CRC, 59 NSCLC). After the baseline FDG-PET, 23 patients (13 CRC, 10 NSCLC) were excluded for several reasons: due to technical issues $(n=7)$, refusal to undergo a second FDG-PET ( $\mathrm{n}=3$ ), death before the second FDG-PET $(\mathrm{n}=3)$ and early discontinuation of chemotherapy due to a significant decline in performance status $(\mathrm{n}=10)$. Therefore, complete datasets of two FDG-PETs were available in 97 patients (48 CRC and 
49 NSCLC) for analysis of therapy response. Patient characteristics are summarised in table 2. No patients were lost during follow-up. Results of survival analysis by Kaplan-Meier are displayed in table 3.

In CRC, mean bodyweight was significantly higher at the baseline scan compared to the follow-up scan $(78.3 \mathrm{~kg}$ versus $77.1 \mathrm{~kg}, \mathrm{p}=0.012$, individual range -15 to $+3 \mathrm{~kg}$ ). Mean serum glucose $\left(5.4 \mathrm{mmol} \cdot \mathrm{L}^{-1}\right.$ versus $5.5 \mathrm{mmol} \cdot \mathrm{L}^{-1}, \mathrm{p}=0.115$, individual range -1.1 to +3.2 $\left.\mathrm{mmol} \cdot \mathrm{L}^{-1}\right)$ and injected dose $(199 \mathrm{MBq}$ versus $204 \mathrm{MBq}, \mathrm{p}=0.592)$ did not differ. In NSCLC, mean serum glucose was significantly higher at the baseline scan compared to the followup scan $\left(5.5 \mathrm{mmol} \cdot \mathrm{L}^{-1}\right.$ versus $5.2 \mathrm{mmol} \cdot \mathrm{L}^{-1}, \mathrm{p}=0.029$, individual range -2.0 to $+2.6 \mathrm{mmol} \cdot \mathrm{L}^{-}$ ${ }^{1}$ ). Mean bodyweight ( $73.9 \mathrm{~kg}$ versus $73.7 \mathrm{~kg}, \mathrm{p}=0.637$, individual range -9.0 to $+10 \mathrm{~kg}$ ) and injected dose (202MBq versus $212 \mathrm{MBq}, \mathrm{p}=0.215)$ did not differ.

\section{Semi-quantitative changes in FDG uptake}

Median interval between baseline and follow-up FDG-PET was 8.9 weeks (IQR 7.0-9.6) in the CRC-group and 7.0 weeks (IQR 5.9-8.6) in the NSCLC-group. Box-and-whisker plots of $\triangle$ SUVs are displayed in figure 1. In both CRC and NSCLC the decline in median of all four SUVs between first and second FDG-PET was statistically significant $(\mathrm{p}<0.001)$. No significant differences between the four compared $\triangle \mathrm{SUVs}$ could be found in CRC $(\mathrm{p}=0.143)$ and NSCLC $(\mathrm{p}=0.059)$.

In $\mathrm{CRC}$ differences in metabolic response between $\Delta \mathrm{SUV}_{\mathrm{LBM}}, \Delta \mathrm{SUV}_{\mathrm{BSA}}$ and $\Delta \mathrm{SUV}_{\mathrm{BW}+\mathrm{G}}$ compared to $\Delta \mathrm{SUV}_{\mathrm{BW}}$ was more than the reproducibility limit in 1,0 and 5 of 48 cases respectively. In NSCLC this was 0,0 and 5 of 49 cases respectively.

\section{Prediction of survival by FDG-PET}

Cox's proportional hazards model for CRC and NSCLC showed high predictive significance for OS and PFS for all four $\triangle \mathrm{SUV}$ normalisations between baseline and follow-up FDG-PET (table 4). 

treatment response as to OS and PFS, patients were dichotomised to the median $\triangle \mathrm{SUV}$. Results are displayed in table 5.

In CRC using a cut-off for $\triangle \mathrm{SUV}$ of $-33 \%$ for $\mathrm{BW}, \mathrm{LBM}$ and $\mathrm{BSA}$ and $-22 \%$ for $\mathrm{BW}+\mathrm{G}$ separated patients who had $90 \% 1$-year OS from those with lower OS rates. In

200 NSCLC these cut-offs were $-56 \%$ for BW, $-57 \%$ for $\mathrm{LBM}$ and BSA and $-53 \%$ for BW+G. The group defined as metabolic responders consisted of $24-31 \%$ of the patients. In contrast to $\mathrm{CRC}$, in NSCLC these numbers were very different from the medians $(\sim-37 \%)$. This prognosis-driven dichotomisation for NSCLC for OS resulted in median ratios of 0.28 (HR 2.8, log rank $\mathrm{p}=0.001)$ for $\Delta \mathrm{SUV}_{\mathrm{BW}}$ and $\Delta \mathrm{SUV}_{\mathrm{BW}+\mathrm{G}}$ and $0.22(\mathrm{HR} 3.1, \log \operatorname{rank} \mathrm{p}<0.001)$ for $\triangle \mathrm{SUV}_{\mathrm{LBM}}$ and for $\triangle \mathrm{SUV}_{\mathrm{BSA}}$.

\section{Discussion}

In this study, we showed for the first time that the method for SUV-normalisation does not influence the predictive value of FDG-PET in CRC and NSCLC in chemotherapy response 210 monitoring. The predictive value of FDG-PET for CRC [6] and NSCLC [19-25] has been studied extensively and is an established predictor for survival. Chemotherapy response monitoring in CRC was performed by different groups, all using absolute [10,26,27] or relative [11] $\mathrm{SUV}_{\mathrm{BW}}$ differences as a measure for change in metabolic activity. In NSCLC, relative changes in SUV as result of therapy have been observed in numerous studies. Some used BW normalised SUV [12,28-30], others used normalisation by BSA and serum glucose [9]. However, for metabolic response assessment none so far investigated which of the different normalisations is optimal as compared to overall and progression free survival.

In both CRC and NSCLC, all four normalised SUVs showed a significant decrease between baseline and follow-up FDG-PET. Although it was shown that bodyweight (in 
220 CRC) and serum glucose (in NSCLC), the major factors for normalisation of SUV, were significantly different between both baseline and follow-up scan, we did not find mutual differences between the four $\Delta \mathrm{SUV}$-normalisations. This suggests that there is no preference for either any of the four normalisations in SUV when therapy response is measured by relative changes in SUVs between baseline and follow-up FDG-PET.

Krak et al. [17] describe that standard deviation between two consecutive scans (made on 2 consecutive days) was $11 \%$ using the same definition of ROI as in this study. Therefore SUVs on 2 consecutive days may vary $\sim \pm 20 \%$ due to reproducibility limits of the test. Using this as a cut-off for a significant different $\Delta \mathrm{SUV}$ between the 2 normalisations we found that especially in $\Delta \mathrm{SUV}_{\mathrm{BW}+\mathrm{G}}$ more often a change in metabolism was found $>20 \%$ different from $\Delta \mathrm{SUV}_{\mathrm{BW}}$. The addition of the extra variable plasma glucose level caused in 5 CRC and 5 NSCLC patients a highly different conclusion about treatment response. To verify the clinical significance of this different magnitude of metabolic response, it was related to survival.

Choosing a cut-off value of metabolic response for 90\% 1-year OS, led to a median survival ratio of 0.22-0.28 for metabolic non-responders versus responders. In CRC the effect of abovementioned cut-off was similar to the median SUV change (and thus selects $\sim 50 \%$ best responders).

We observed higher hazard ratios for NSCLC than CRC. This suggests that similar reduction in SUV in CRC has less effect on patient overall survival than in NSCLC. This may be caused by biological differences and differences in chemosensitivity of both types of cancer. However, changes in glucose metabolic rate, are not only dependent on biological behaviour of the tumour to the given treatment, but are also dependent on the treatment protocol and the timing of follow-up scanning. Both these biological differences and variation in treatment and follow-up protocol hinder determination of optimal cut-offs.

245 Therefore standardisation of response measurement protocols are necessary and cut-offs should be dependent of tumour-type, antitumour treatment and timing of evaluation. 
Earlier publications $[11,12]$ showed that SUV is a sufficiently robust measure for treatment response monitoring and can reliably replace more complex, invasive and timeconsuming measures such as Patlak analysis which determines glucose metabolic rate 250 (MR $\left.\mathrm{glc}_{\mathrm{c}}\right)$. Apart from being a less time-consuming method, SUV can be calculated from a whole-body FDG-PET study including all metastatic lesions. Furthermore, no input function is required for SUV-determination. The advantage of relative SUV-determination compared to absolute SUVs, however, is that it is easier to combine data from different studies because they are less sensitive to introduction of errors due to noise, image resolution and ROI-definition compared to absolute SUVs [17,31-33]. If the patient is scanned in the same hospital on the same scanner, some scanner related factors and patient related factors can be ignored [33].

Different normalisation methods for absolute SUV values have been addressed by others [34-38], reporting varying results. Some have shown that SUV corrected for BSA 260 proved to be more accurate, in adult [34] and paediatric [38] patients, compared to the gold standard $\mathrm{MR}_{\mathrm{glc}}$. It was suggested that normalisation is necessary because of variability in SUV due to body composition and habitus as well as plasma glucose [32,39]. Menda et al. [36] observed no advantage of any SUV correction for accuracy of diagnosis of pulmonary malignancy. In contrast with our study, these studies did not address therapy response, and therefore did not take into consideration the measurement of relative changes in SUV.

Krak et al. [35] compared different normalisations for relative SUV change in 20 women with locally advanced or metastasised breast cancer with the golden standard of non-linear regression after 1, 2 or 6 courses of chemotherapy. They concluded that of the investigated normalisation methods, relative change of SUV corrected for both LBM and glucose showed highest correlation with relative change in glucose metabolic rate as calculated by non-linear regression. This study did not correlate SUV corrections with clinical outcome parameters such as patient survival. 
Stahl et al. [37] compared histological response in 43 patients with locally advanced gastric carcinomas with the same four normalisations for relative SUV change as in our study, between baseline and follow-up FDG-PET (after 2 weeks, during first cycle of platinum-based chemotherapy). They, too, concluded that no normalisation method of $\Delta$ SUVs has an advantage for response prediction and that the theoretical benefits of the measures to reduce the dependency of the SUV on BW or plasma glucose do not translate into a detectable clinical benefit.

Our study showed no preference of any SUV-normalisation in therapy response monitoring in CRC and NSCLC. This could be caused by the fact that SUVs used in therapy response monitoring are compared in individual patients and not between different patients. It can be easily derived that an increase in bodyweight alone of $10 \%$, increases $\Delta \mathrm{SUV}_{\mathrm{BW}}$ and $\Delta \mathrm{SUV}_{\mathrm{BW}+\mathrm{G}}$ by $10 \%, \Delta \mathrm{SUV}_{\mathrm{BSA}}$ by $4.1 \%\left(=[1.10]^{0.425}-1\right)$ and has no effect on $285 \Delta \mathrm{SUV}_{\mathrm{LBM}}$. An increase of plasma glucose alone of $10 \%$ increases $\Delta \mathrm{SUV}_{\mathrm{BW}+\mathrm{G}}$ by $10 \%$. The effect of chemotherapy and disease on body composition seems to be relatively small. Therefore it is possible to select the SUV, which is simplest, without introduction of extra parameters like bodyweight, length or serum glucose. All these parameters necessitate calibration and may for that reason potentially introduce extra uncertainties. Moreover, simplifying FDG-PET methodology in therapy response monitoring could facilitate its introduction in routine clinical practice.

The variety of applied analytical methods is vast, which hampers multicentre research. Clear methods for standardisation of acquisition, reconstruction, ROIdetermination and SUV-normalisation need to be determined [33]. In fact, methodology of metabolic response evaluation needs to be standardised in evidence based multidisciplinary international guidelines. This has been attempted previously by the European Organisation for Research and Treatment of Cancer (EORTC) [40] and the National Cancer Institute (NCI) [15]. However, they provide consensus-based recommendations, rather than advice- 
based on scientific proof. Evidence-based guidelines would be of utmost importance for the

300 interpretation and comparison of multicentre trials.

\section{Conclusion}

In chemotherapy response evaluation in both CRC and patients with NSCLC, relative SUV changes in FDG-PET have high predictive value for patient survival. Using relative changes in tumour FDG-uptake for a patient, no normalisation for body habitus in SUVcalculation seems superior, since all perform equally well for prediction of survival in both types of cancer. Therefore theoretical advantages of one normalisation method over another, do not translate into clinical relevant changes. This finding in combination with the fact that relative (rather than absolute) changes are less dependent of quantitative PET

310 acquisition protocols [17,31], will facilitate determination of response to therapy. This could be a step towards standardizing the results of therapy monitoring which might be used for pooling of data in multicentre trials. Moreover, it may enable integration of metabolic response measurement in the development of clinical guidelines by giving evidence-based definitions of partial response, stable disease and progressive disease for different cancer types. 


\section{Reference List}

1. Herbertson, RA, Lee, ST, Tebbutt, N, Scott, AM. The expanding role of PET technology in the management of patients with colorectal cancer. Ann Oncol 2007; 18:1774-1781.

2. Valk, PE, Abella-Columna, E, Haseman, MK, Pounds, TR, Tesar, RD, Myers, RW, et al. Whole-body PET imaging with [18F]fluorodeoxyglucose in management of recurrent colorectal cancer. Arch Surg 1999; 134:503-511; discussion 511-503.

3. Watson, AJ, Lolohea, S, Robertson, GM, Frizelle, FA. The role of positron emission tomography in the management of recurrent colorectal cancer: a review. Dis Colon Rectum 2007; 50:102-114.

4. Mac Manus, MP, Hicks, RJ, Matthews, JP, McKenzie, A, Rischin, D, Salminen, EK, et al. Positron emission tomography is superior to computed tomography scanning for response-assessment after radical radiotherapy or chemoradiotherapy in patients with non-small-cell lung cancer. J Clin Oncol 2003; 21:1285-1292.

5. Vansteenkiste, J, Fischer, BM, Dooms, C, Mortensen, J. Positron-emission tomography in prognostic and therapeutic assessment of lung cancer: systematic review. Lancet Oncol 2004; 5:531-540.

6. de Geus-Oei, LF, Wiering, B, Krabbe, PF, Ruers, TJ, Punt, CJ, Oyen, WJ. FDG-PET for prediction of survival of patients with metastatic colorectal carcinoma. Ann Oncol 2006; 17:1650-1655.

7. de Geus-Oei, LF, van der Heijden, HF, Corstens, FH, Oyen, WJ. Predictive and prognostic value of FDG-PET in nonsmall-cell lung cancer: a systematic review. Cancer 2007; 110:1654-1664.

8. Dimitrakopoulou-Strauss, A, Hoffmann, M, Bergner, R, Uppenkamp, M, Eisenhut, M, Pan, L, et al. Prediction of short-term survival in patients with advanced nonsmall cell lung cancer following chemotherapy based on 2-deoxy-2-[F-18]fluoro-D-glucose-positron emission tomography: a feasibility study. Mol Imaging Biol 2007; 9:308-317.

9. Hoekstra, CJ, Stroobants, SG, Smit, EF, Vansteenkiste, J, van Tinteren, H, Postmus, PE, et al. Prognostic relevance of response evaluation using [18F]-2-fluoro-2-deoxy-D-glucose positron emission tomography in patients with locally advanced non-small-cell lung cancer. J Clin Oncol 2005; 23:8362-8370.

10. Dimitrakopoulou-Strauss, A, Strauss, LG, Rudi, J. PET-FDG as predictor of therapy response in patients with colorectal carcinoma. $Q J \mathrm{Nucl} \mathrm{Med}$ 2003; 47:8-13.

11. de Geus-Oei, LF, van Laarhoven, HW, Visser, EP, Hermsen, R, van Hoorn, BA, Kamm, YJ, et al. Chemotherapy response evaluation with FDG-PET in patients with colorectal cancer. Ann Oncol 2008; 19:348-352.

12. de Geus-Oei, LF, van der Heijden, HF, Visser, EP, Hermsen, R, van Hoorn, BA, Timmer-Bonte, JN, et al. Chemotherapy response evaluation with 18 F-FDG PET in patients with non-small cell lung cancer. $J$ Nucl Med 2007; 48:1592-1598. 
13. Hoekstra, CJ, Hoekstra, OS, Stroobants, SG, Vansteenkiste, J, Nuyts, J, Smit, EF, et al. Methods to monitor response to chemotherapy in non-small cell lung cancer with 18F-FDG PET. J Nucl Med 2002; 43:1304-1309.

14. Hoekstra, CJ, Paglianiti, I, Hoekstra, OS, Smit, EF, Postmus, PE, Teule, GJ, et al. Monitoring response to therapy in cancer using [18F]-2-fluoro-2deoxy-D-glucose and positron emission tomography: an overview of different analytical methods. Eur J Nucl Med 2000; 27:731-743.

15. Shankar, LK, Hoffman, JM, Bacharach, S, Graham, MM, Karp, J, Lammertsma, AA, et al. Consensus recommendations for the use of $18 \mathrm{~F}$ FDG PET as an indicator of therapeutic response in patients in National Cancer Institute Trials. J Nucl Med 2006; 47:1059-1066.

16. Therasse, P, Arbuck, SG, Eisenhauer, EA, Wanders, J, Kaplan, RS, Rubinstein, L, et al. New guidelines to evaluate the response to treatment in solid tumors. European Organization for Research and Treatment of Cancer, National Cancer Institute of the United States, National Cancer Institute of Canada. J Natl Cancer Inst 2000; 92:205-216.

17. Krak, NC, Boellaard, R, Hoekstra, OS, Twisk, JW, Hoekstra, CJ, Lammertsma, AA. Effects of ROI definition and reconstruction method on quantitative outcome and applicability in a response monitoring trial. Eur $J$ Nucl Med Mol Imaging 2005; 32:294-301.

18. Nahmias, C, Wahl, LM. Reproducibility of standardized uptake value measurements determined by $18 \mathrm{~F}-\mathrm{FDG}$ PET in malignant tumors. $\mathrm{J} \mathrm{Nucl}$ Med 2008; 49:1804-1808.

19. Ahuja, V, Coleman, RE, Herndon, J, Patz, EF, Jr. The prognostic significance of fluorodeoxyglucose positron emission tomography imaging for patients with nonsmall cell lung carcinoma. Cancer 1998; 83:918-924.

20. Downey, RJ, Akhurst, T, Gonen, M, Vincent, A, Bains, MS, Larson, S, et al. Preoperative F-18 fluorodeoxyglucose-positron emission tomography maximal standardized uptake value predicts survival after lung cancer resection. J Clin Oncol 2004; 22:3255-3260.

21. Dhital, K, Saunders, CA, Seed, PT, O'Doherty, MJ, Dussek, J. [(18)F]Fluorodeoxyglucose positron emission tomography and its prognostic value in lung cancer. Eur J Cardiothorac Surg 2000; 18:425428.

22. Vansteenkiste, JF, Stroobants, SG, Dupont, PJ, De Leyn, PR, Verbeken, EK, Deneffe, GJ, et al. Prognostic importance of the standardized uptake value on (18)F-fluoro-2-deoxy-glucose-positron emission tomography scan in non-small-cell lung cancer: An analysis of 125 cases. Leuven Lung Cancer Group. J Clin Oncol 1999; 17:3201-3206.

23. Jeong, HJ, Min, JJ, Park, JM, Chung, JK, Kim, BT, Jeong, JM, et al. Determination of the prognostic value of [(18)F]fluorodeoxyglucose uptake by using positron emission tomography in patients with non-small cell lung cancer. Nucl Med Commun 2002; 23:865-870.

24. Higashi, K, Ueda, Y, Arisaka, Y, Sakuma, T, Nambu, Y, Oguchi, M, et al. 18F-FDG uptake as a biologic prognostic factor for recurrence in patients with surgically resected non-small cell lung cancer. J Nucl Med 2002; 43:39-45.

25. Sasaki, R, Komaki, R, Macapinlac, H, Erasmus, J, Allen, P, Forster, K, et al. [18F]fluorodeoxyglucose uptake by positron emission tomography 
predicts outcome of non-small-cell lung cancer. J Clin Oncol 2005; 23:1136-1143.

26. Findlay, M, Young, H, Cunningham, D, Iveson, A, Cronin, B, Hickish, T, et al. Noninvasive monitoring of tumor metabolism using fluorodeoxyglucose and positron emission tomography in colorectal cancer liver metastases: correlation with tumor response to fluorouracil. $J$ Clin Oncol 1996; 14:700-708.

27. Dimitrakopoulou-Strauss, A, Strauss, LG, Burger, C, Ruhl, A, Irngartinger, G, Stremmel, W, et al. Prognostic aspects of 18F-FDG PET kinetics in patients with metastatic colorectal carcinoma receiving FOLFOX chemotherapy. J Nucl Med 2004; 45:1480-1487.

28. Vansteenkiste, JF, Stroobants, SG, De Leyn, PR, Dupont, PJ, Verbeken, EK. Potential use of FDG-PET scan after induction chemotherapy in surgically staged IIIa-N2 non-small-cell lung cancer: a prospective pilot study. The Leuven Lung Cancer Group. Ann Oncol 1998; 9:1 193-1198.

29. Pottgen, C, Levegrun, S, Theegarten, D, Marnitz, S, Grehl, S, Pink, R, et al. Value of 18F-fluoro-2-deoxy-D-glucose-positron emission tomography/computed tomography in non-small-cell lung cancer for prediction of pathologic response and times to relapse after neoadjuvant chemoradiotherapy. Clin Cancer Res 2006; 12:97-106.

30. Weber, WA, Petersen, V, Schmidt, B, Tyndale-Hines, L, Link, T, Peschel, $\mathrm{C}$, et al. Positron emission tomography in non-small-cell lung cancer: prediction of response to chemotherapy by quantitative assessment of glucose use. J Clin Oncol 2003; 21:2651-2657.

31. Boellaard, R, Krak, NC, Hoekstra, OS, Lammertsma, AA. Effects of noise, image resolution, and ROI definition on the accuracy of standard uptake values: a simulation study. J Nucl Med 2004; 45:1519-1527.

32. Keyes, JW, Jr. SUV: standard uptake or silly useless value? J Nucl Med 1995; 36:1836-1839.

33. Westerterp, M, Pruim, J, Oyen, W, Hoekstra, O, Paans, A, Visser, E, et al. Quantification of FDG PET studies using standardised uptake values in multi-centre trials: effects of image reconstruction, resolution and ROI definition parameters. Eur J Nucl Med Mol Imaging 2007; 34:392-404.

34. Graham, MM, Peterson, LM, Hayward, RM. Comparison of simplified quantitative analyses of FDG uptake. Nucl Med Biol 2000; 27:647-655.

35. Krak, NC, van der Hoeven, JJ, Hoekstra, OS, Twisk, JW, van der Wall, E, Lammertsma, AA. Measuring [(18)F]FDG uptake in breast cancer during chemotherapy: comparison of analytical methods. Eur J Nucl Med Mol Imaging 2003; 30:674-681.

36. Menda, Y, Bushnell, DL, Madsen, MT, McLaughlin, K, Kahn, D, Kernstine, KH. Evaluation of various corrections to the standardized uptake value for diagnosis of pulmonary malignancy. Nucl Med Commun 2001; 22:1077-1081.

37. Stahl, A, Ott, K, Schwaiger, M, Weber, WA. Comparison of different SUV-based methods for monitoring cytotoxic therapy with FDG PET. Eur J Nucl Med Mol Imaging 2004; 31:1471-1478.

38. Yeung, HW, Sanches, A, Squire, OD, Macapinlac, HA, Larson, SM, Erdi, YE. Standardized uptake value in pediatric patients: an investigation to determine the optimum measurement parameter. Eur J Nucl Med Mol Imaging 2002; 29:61-66. 
39. Hamberg, LM, Hunter, GJ, Alpert, NM, Choi, NC, Babich, JW, Fischman, AJ. The dose uptake ratio as an index of glucose metabolism: useful parameter or oversimplification? J Nucl Med 1994; 35:1308-1312.

40. Young, H, Baum, R, Cremerius, U, Herholz, K, Hoekstra, O, Lammertsma, $\mathrm{AA}$, et al. Measurement of clinical and subclinical tumour response using [18F]-fluorodeoxyglucose and positron emission tomography: review and 1999 EORTC recommendations. European Organization for Research and Treatment of Cancer (EORTC) PET Study Group. Eur J Cancer 1999; 35:1773-1782. 


\section{Tables}

\begin{tabular}{|c|c|}
\hline SUV normalisation: & Formula: \\
\hline SUV $_{\text {BW }}$ (bodyweight) & $\mathrm{SUV}_{\mathrm{BW}}=\mathrm{C}_{\mathrm{t}} \cdot\left(\mathrm{I} \cdot \mathrm{BW}^{-1}\right)^{-1}$ \\
\hline \multirow[t]{3}{*}{ SUV LBM (lean body mass) } & $\mathrm{SUV}_{\mathrm{LBM}}=\mathrm{C}_{\mathrm{t}} \cdot\left(\mathrm{I} \cdot \mathrm{LBM}^{-1}\right)^{-1}$ \\
\hline & Male: $\quad \mathrm{LBM}=48.0+1.06 \cdot(\mathrm{h}-152)$ \\
\hline & Female: $\mathrm{LBM}=45.5+0.91 \cdot(\mathrm{h}-152)$ \\
\hline \multirow[t]{2}{*}{ SUV $V_{\text {BSA }}($ body surface area) } & $\mathrm{SUV}_{\mathrm{BSA}}=\mathrm{C}_{\mathrm{t}} \cdot\left(\mathrm{I} \cdot \mathrm{BSA}^{-1}\right)^{-1}$ \\
\hline & $\mathrm{BSA}=0.007184 \cdot \mathrm{BW}^{0.425} \cdot \mathrm{h}^{0.725}$ \\
\hline SUV $_{B W+G}($ bodyweight and plasma glucose) & $\mathrm{SUV}_{\mathrm{BW}+\mathrm{G}}=\mathrm{SUV}_{\mathrm{BW}} \cdot \mathrm{PG}$ \\
\hline
\end{tabular}

Table 1: Four SUV normalisations evaluated in this study. $C_{t}$ : tissue concentration $\left[\mathrm{kBq} \cdot \mathrm{ml}^{-1}\right]$; I: injected dose $[\mathrm{MBq}]$; BW: bodyweight [kg]; LBM: lean body mass [kg]; $\mathrm{h}$ : height $[\mathrm{cm}]$; BSA: body surface area $\left[\mathrm{m}^{2}\right]$; PG: plasma glucose $\left[\mathrm{mmol} . \mathrm{L}^{-1}\right]$. 


\begin{tabular}{|c|c|c|}
\hline Characteristics: & CRC: & NSCLC: \\
\hline \multicolumn{3}{|l|}{ Demography: } \\
\hline Number of patients & 48 & 49 \\
\hline Mean age (year) [range] & $61.0[44.7-78.9]$ & $59.6[38.5-76.2]$ \\
\hline Men (\%) & 73 & 74 \\
\hline \multicolumn{3}{|l|}{ Location of metastases (\%): } \\
\hline Liver & 86 & 14 \\
\hline Lung & 29 & 37 \\
\hline Lymph nodes & 8 & 67 \\
\hline Bone & 4 & 12 \\
\hline Brain & - & 16 \\
\hline Adrenals & - & 12 \\
\hline Other & - & 10 \\
\hline None & - & 6 \\
\hline \multicolumn{3}{|l|}{ Histology (\%): } \\
\hline Adenocarcinoma & 94 & 45 \\
\hline Squamous cell carcinoma & - & 43 \\
\hline Large cell carcinoma & - & 8 \\
\hline Mucinous adenocarcinoma & 6 & - \\
\hline Bronchoalveolar cell carcinoma & - & 2 \\
\hline Clear cell carcinoma & - & 2 \\
\hline \multicolumn{3}{|l|}{ Tumour grade (\%): } \\
\hline Undifferentiated: & 2 & - \\
\hline Very poor & 4 & 27 \\
\hline Poor & 10 & 12 \\
\hline Intermediate & 60 & 12 \\
\hline
\end{tabular}




\begin{tabular}{lll}
\hline Well & 4 & 2 \\
Mucinous & - & 2 \\
Unspecified & 19 & 45 \\
\hline Tumour stage (\%): & - & $2(\mathrm{~B})$ \\
I & - & $2(\mathrm{~A})$ \\
II & - & $16(\mathrm{~A}), 20$ (B) \\
III & 100 & 59 \\
IV & & \\
\hline
\end{tabular}

Table 2: Patient characteristics. CRC: colorectal carcinoma; NSCLC: non-small cell lung carcinoma; N/a: not applicable. 


\begin{tabular}{llllll}
\hline & $\begin{array}{l}\text { Event-free } \\
\mathbf{n}[\%]\end{array}$ & $\begin{array}{l}\text { Median [CI] } \\
\mathbf{( w e e k s )}\end{array}$ & $\begin{array}{l}\mathbf{1} \text { year [CI] } \\
\mathbf{( \% )}\end{array}$ & $\begin{array}{l}\mathbf{2} \text { year[CI] } \\
\mathbf{( \% )}\end{array}$ & $\begin{array}{l}\text { 3 year[CI] } \\
\mathbf{( \% )}\end{array}$ \\
\hline CRC: & & & & & \\
Overall survival & $7[15]$ & $85[70-100]$ & $77[65-89]$ & $34[21-48]$ & $14[3-25]$ \\
Progression free survival & $1[2]$ & $24[19-29]$ & $17[6-27]$ & $4[0-10]$ & $2[0-6]$ \\
NSCLC: & & & & & \\
Overall survival & $7[14]$ & $62[42-82]$ & $57[43-71]$ & $39[25-53]$ & $22[10-34]$ \\
Progression free survival & $4[8]$ & $30[17-42]$ & $30[17-42]$ & $19[8-30]$ & $10[1-18]$
\end{tabular}

Table 3: Results of follow-up. CRC: colorectal carcinoma; NSCLC: non-small cell lung carcinoma; CI: 95\%confidence interval. 


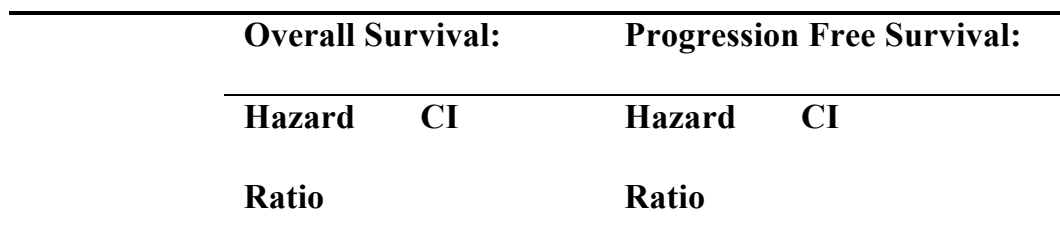

\begin{tabular}{lcccc}
\hline $\mathbf{C R C}:$ & & & & \\
\hline$\Delta \mathbf{S U V}$ & & & & \\
$\mathbf{B W}$ & $1.16 \dagger$ & $1.04-1.29$ & $1.15 \dagger$ & $1.04-1.28$ \\
$\mathbf{S} \mathbf{S U V}$ & $1.14 \dagger$ & $1.04-1.26$ & $1.15 \dagger$ & $1.04-1.27$ \\
$\mathbf{\Delta S U M}$ & & $1.04-1.27$ & $1.15 \dagger$ & $1.04-1.27$ \\
$\mathbf{\Delta S U V}$ & $1.15 \dagger$ & $1.03-1.24$ & $1.15 \dagger$ & $1.04-1.26$
\end{tabular}

\begin{tabular}{lcccc}
\hline NSCLC: & & & & \\
\hline$\Delta \mathbf{S U V}$ & & & & \\
& $1.12 \dagger$ & $1.03-1.20$ & $1.12 \dagger$ & $1.04-1.20$ \\
$\Delta \mathbf{S U W}$ & $1.10 \dagger$ & $1.03-1.17$ & $1.10 \dagger$ & $1.04-1.18$ \\
$\mathbf{\Delta S U M}$ & $1.10 \dagger$ & $1.03-1.18$ & $1.12 \dagger$ & $1.04-1.18$ \\
$\mathbf{\Delta S U S A}$ & $1.13 \dagger$ & $1.04-1.21$ & $1.12 \dagger$ & $1.03-1.20$
\end{tabular}

Table 4: Results of univariate Cox proportional hazards regression analysis for overall and progression free survival using $\triangle \mathrm{SUV}$ s between the scan at baseline and evaluation. $\triangle \mathrm{SUV}$ : relative change in Standardised uptake value between baseline and follow-up; CRC: colorectal carcinoma; NSCLC: non-small cell lung carcinoma; BW: normalisation for bodyweight; LBM: normalisation for lean body mass; BSA: normalised for body surface area; $\mathrm{BW}+\mathrm{G}$ : normalised for bodyweight and plasma glucose; CI: 95\%-confidence interval; *per ten percent change; †Significant by Wald's $\chi^{2}$ test. 


\begin{tabular}{|c|c|c|c|c|c|c|}
\hline & \multicolumn{3}{|c|}{ Overall Survival: } & \multicolumn{3}{|c|}{ Progression Free Survival: } \\
\hline & Median & Hazard & CI & Median & Hazard & CI \\
\hline & Ratio & Ratio & & Ratio & Ratio & \\
\hline \multicolumn{7}{|l|}{ CRC: } \\
\hline$\Delta \mathbf{S U V} \mathbf{V}_{\mathbf{B W}}$ & 0.60 & $1.95 \dagger$ & $1.03-3.69$ & 0.59 & $1.92 \dagger$ & $1.07-3.44$ \\
\hline$\Delta \mathbf{S U V} \mathbf{V}_{\mathbf{L B M}}$ & 0.82 & 1.33 & $0.71-2.47$ & 0.65 & 1.57 & $0.88-2.80$ \\
\hline$\Delta \mathbf{S U V} \mathbf{V}_{\text {BSA }}$ & 0.60 & $1.95 \dagger$ & $1.03-3.69$ & 0.59 & $1.92 \dagger$ & $1.07-3.44$ \\
\hline$\Delta \mathbf{S U V} \mathbf{V}_{\mathbf{B W}+\mathbf{G}}$ & 0.60 & $2.48 \dagger$ & $1.26-4.89$ & 0.52 & $2.08 \dagger$ & $1.16-3.74$ \\
\hline \multicolumn{7}{|l|}{ NSCLC: } \\
\hline$\Delta S U V_{B W}$ & 0.48 & $2.12 \dagger$ & $1.14-3.92$ & 0.37 & $2.97 \dagger$ & $1.56-5.66$ \\
\hline$\Delta S U V_{\text {LBM }}$ & 0.48 & $2.12 \dagger$ & $1.14-3.92$ & 0.37 & $2.97 \dagger$ & $1.56-5.66$ \\
\hline$\Delta S U V_{B S A}$ & 0.48 & $2.12 \dagger$ & $1.14-3.92$ & 0.37 & $2.97 \dagger$ & $1.56-5.66$ \\
\hline$\Delta \mathbf{S U V} \mathbf{V}_{\mathbf{B W}+\mathbf{G}}$ & 0.70 & 1.48 & $0.80-2.74$ & 0.45 & 1.60 & $0.89-2.88$ \\
\hline
\end{tabular}

Table 5: Results of univariate Kaplan-Meier analysis for overall and progression free survival using $\triangle$ SUVs between the scan at baseline and evaluation dichotomised at their median for response or non-response. $\triangle \mathrm{SUV}$ : relative change in Standardised uptake value between baseline and follow-up scan; CRC: Colorectal carcinoma; NSCLC: Non-small cell lung carcinoma; BW: normalisation for bodyweight; LBM: normalisation for lean body mass; BSA: normalised for body surface area; $\mathrm{BW}+\mathrm{G}$ : normalised for bodyweight and plasma glucose; CI: 95\%-Confidence Interval; $\uparrow$ Significant by log rank test. 
Figures

CRC:

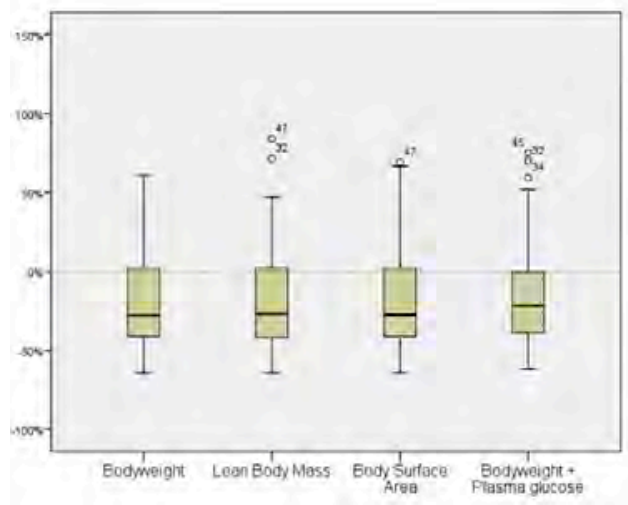

NSCLC:

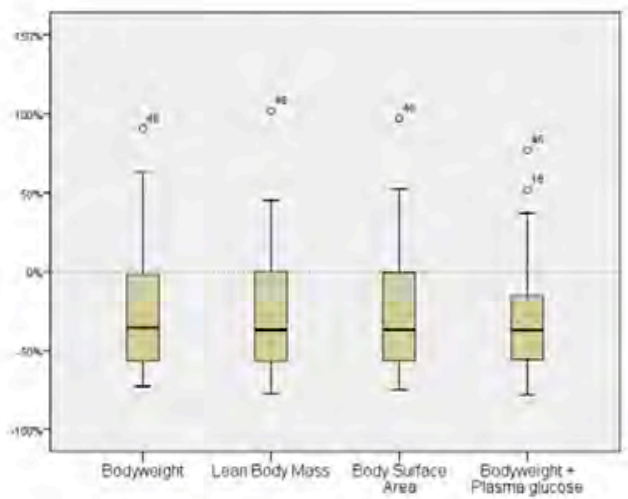

Figure 1: Box-and-whisker plots of four normalisations for percentage change in Standardised Uptake Value between baseline and follow-up ${ }^{18}$ F-2-fluoro-2-deoxy-Dglucose positron emission tomography. The boxes are constructed by the mean, first and third quartiles. The whiskers extend 1.5 times the interquartile range above and below the 25th and 75 th percentiles. Outliers are displayed as open circles. CRC: colorectal carcinoma; NSCLC: non-small cell lung carcinoma. 